\title{
Association of Hyperthyroidism and Thyroid Autoantibodies with Moyamoya Disease and Its Stroke Event: A Population-based Case-control Study and Meta-analysis
}

\author{
Jun Hyong AhN, ${ }^{1^{*}}$ Jin Pyeong JeON,,$^{1^{*}}$ Jeong Eun KIM, ${ }^{2}$ Eun Jin HA, ${ }^{2}$ \\ Won-Sang CHO, ${ }^{2}$ Young Joo PARK, ${ }^{3}$ Nam Han CHO, ${ }^{4}$ \\ Hoon Sung CHOI, ${ }^{5}$ Hyun-Seung KANG, ${ }^{2}$ Young-Je SON, ${ }^{2}$ \\ Jae Seung BANG, ${ }^{2}$ and Chang Wan $\mathrm{OH}^{2}$
}

\begin{abstract}
${ }^{1}$ Department of Neurosurgery, Hallym University College of Medicine, Chuncheon, Korea;
${ }^{2}$ Department of Neurosurgery, Seoul National University College of Medicine, Seoul, Korea;

${ }^{3}$ Department of Internal Medicine, Seoul National University College of Medicine, Seoul, Korea;

${ }^{4}$ Department of Preventive Medicine, Ajou University College of Medicine, Suwon, Korea;

${ }^{5}$ Department of Internal Medicine, Kangwon National University College of Medicine, Chuncheon, Korea
\end{abstract}

\begin{abstract}
The aim of this study was to investigate the specific thyroid condition and thyroid autoantibodies in adult moyamoya disease (MMD) according to clinical presentation (ischemia vs. hemorrhage stroke). In addition, a meta-analysis was performed to reveal the association between adult MMD and elevated thyroid function, or autoantibodies. Prospectively collected data on 169 consecutive patients with MMD at a single institution were analyzed. Community-based controls matched for age and sex were selected for comparison. Penalized multinomial logistic regression analysis was used for factors affecting stroke. For meta-analysis, heterogeneity was evaluated by using the $I^{2}$ test. If $I^{2}<50 \%$, a fixed effect model was used. Fifty-four cases $(32.0 \%)$ presented with ischemic stroke and 37 cases $(21.9 \%)$ with hemorrhage stroke. Hyperthyroidism had a marginally increased risk of MMD with ischemic stroke with reference value of MMD without stroke [odds ratio (OR), $2.53 ; P=0.055$ ]. Anti-thyroperoxidase antibody (TPOAb) increased the risk of MMD presenting with ischemic stroke significantly $(\mathrm{OR}, 2.99 ; P=0.020)$. A meta-analysis revealed that adult MMD was significantly associated with elevated autoantibodies $(\mathrm{OR}, 7.663 ; P=0.002)$ and hyperthyroidism $(O R, 10.936 ; P<0.001)$. Elevated TPOAb and hyperthyroidism may play important roles in adult MMD with ischemic stroke. Studies focusing on targeted hyperthyroidism and thyroid autoantibodies are necessary in treating adult MMD patients in the future.
\end{abstract}

Key words: moyamoya disease, stroke, thyroid

\section{Introduction}

Typical moyamoya disease (MMD) is referred as an abnormal bilateral progressive steno-occlusive disorder of unknown etiologies at the distal internal

Received October 18, 2017; Accepted November 28, 2017

Copyright $@ 2018$ by The Japan Neurosurgical Society This work is licensed under a Creative Commons AttributionNonCommercial-NoDerivatives International License.

*Jun Hyong Ahn and Jin Pyeong Jeon equally distributed to this work. carotid artery (ICA) or proximal middle cerebral artery (MCA) and anterior cerebral artery (ACA) with the formation of abnormal collateral vessels. ${ }^{1)}$ Although many studies have been performed to reveal the pathogenesis of MMD, exact mechanism still remains unclear. After coexistence of MMD and Grave's disease had been published, ${ }^{2)}$ associations between MMD and thyroid functional status or thyroid autoimmunity have been increasingly published. ${ }^{3,4)}$ MMD with Graves disease or hyperthyroidism is much more common among East Asian population, while association of Hashimoto 
disease with MMD is more common among Caucasian patients. ${ }^{5,6)} \mathrm{Kim}$ et al. $^{7)}$ reported that elevated thyroid autoantibodies [odds ratio (OR): 4.87; 95\% confidence initial (CI): 1.588-15.277] and smoking were related to MMD compared to non-MMD stroke. Li et al. ${ }^{3)}$ also demonstrated higher level of thyroid autoantibodies and thyroid function in pediatric MMD. A recent meta-analysis ${ }^{8)}$ showed that elevated thyroid autoantibodies (OR: 8.77; $P<$ 0.001 ) and thyroid function (OR: 9.74; $P=0.003$ ) were significantly associated with MMD development after reviewing two adults and one pediatric MMD studies. ${ }^{7-9)}$ Nevertheless, heterogeneity among studies such as patients' age and inclusion criteria could be concerns to the interpretation. MMD prevalence shows bimodal age pattern. ${ }^{10)}$ Compared to pediatric MMD, hemorrhagic presentation was more evident in adult MMD, especially in Asia. ${ }^{10-12)}$ An association between thyroid function status and their clinical presentation (cerebral ischemia vs. intracranial hemorrhage) was not also properly evaluated. Moreover, some results were obtained by comparing hospital-based controls without matched for age and sex..$^{7,8)}$

Therefore, we investigated the specific thyroid condition and thyroid autoantibodies in adult MMD according to their clinical presentation using community-based controls. In addition, we conducted a comprehensive meta-analysis to assess the association between thyroid abnormalities and MMD in adults.

\section{Methods}

\section{Patients}

Prospectively collected data on 169 consecutive patients with MMD were analyzed. Of these, the number of patients with newly diagnosed MMD was 59. Six patients with overt hyperthyroidism prescribed methimazole. For control groups, age- and sex- matched healthy controls were selected from the Korean rural community of Ansung. This study included a biennial examination of the participants who were recruited for the Korean Health and Genome Study (KHGS) in 2001. ${ }^{13)}$ Clinical informations such as presenting symptoms, age, gender, hypertension, diabetes, hyperlipidemia, coronary heart disease and smoking were reviewed. Levels of serum triiodothyronine (T3), free thyroxine (T4), thyrotropin (TSH), anti-thyroperoxidase antibody (TPOAb), anti-thyroglobulin antibody (TGAb) and thyrotropin receptor antibody (TRAb) were measured. Thyroid function status was divided into 3 groups of euthyroid, hypothyroid and hyperthyroid status according to based on previous studies. ${ }^{8,14)}$ All patients provided written informed consent, and the study protocol was approved by the Institutional Review Boards.

\section{Literature search and data abstraction for meta-analysis}

From 1966 to December 2016, an electronic search through PubMed, Embase, and the Cochrane Central Register of Controlled trials in the Cochrane Library were performed using the key words "moyamoya disease", "thyroid function", "thyroid autoantibody", "thyroid", "adult moyamoya disease" and "hyperthyroidism". ${ }^{8)}$ Our inclusion criteria were: (1) adult MMD patients over 16 years of age;15) (2) typical bilateral or probable MMD; (3) clear information on thyroid autoantibodies and thyroid function; (4) clinical studies included more than 10 patients; and (5) prospective or retrospective case-controlled studies which had quality score over 5 on the NewcastleOttawa scale (NOS). ${ }^{8}$ The exclusion criteria were: (1) incomplete data; (2) review articles or case reports, (3) study not written in English, and (4) secondary ICA stenosis or occlusion due to atherosclerosis, trauma or radiation. ${ }^{16)}$ Two authors independently evaluated the eligibility of the studies and extracted the data using a uniform standardized form. Disagreements between two authors were resolved by discussion and consultation with a third author. The primary endpoint was to assess an association between adult MMD and elevated thyroid function, or autoantibodies. This meta-analysis was performed according to the Preferred Reporting items for Systematic Reviews and Meta-analyses (PRISMA) guidelines.

\section{Statistical analysis}

For the comparison of variables in MMD according to presentation, analysis of variance and KruskalWallis test, or chi-square and fisher's exact test were used for continuous and categorical data, respectively. Multinomial logistic regression analysis was used to evaluate the factors affecting ischemic or hemorrhage stroke, with MMD without stroke as the reference value. To solve the problems of separation, penalized multinomial logistic regression analysis were employed. The effects of thyroid function status and thyroid autoantibodies on stroke were assessed separately in model 1 and 2 to address the multicollinearity between these two variables. Regarding meta-analysis, the cumulative incidence (event rate) and 95\% CI were estimated from each study. Dichotomous variables are presented as OR with a $95 \%$ CI. Heterogeneity was evaluated by using the $I^{2}$ test. If $I^{2}<50 \%$, a fixed effect model was used. ${ }^{17)}$ Publication bias was determined using funnel plot, Egger's test of the intercept, and the Begg and Mazumdar rank correlation test. ${ }^{18)}$ SAS, 
version 9.3 (SAS Institute, Cary, North Carolina), R software V3.00 and comprehensive meta-analysis (CMA) software (CMA v2.2.064; Biostat, Englewood, NJ) were utilized for all the above, with statistical significance indicated at $P<0.05$.

\section{Results}

\section{Association of adult MMD with thyroid function and autoantibodies}

To know the differences in the level of thyroid function and autoantibodies between MMD and controls, age- and sex-matched MMD and controls of Ansung cohort were selected. Baseline characteristics between the two groups are described in Table 1 . Increased free T4 and decreased TSH were measured compared to controls. Hyperthyroidism $(10.3 \%$ vs. $1.3 \%$ in controls; $P<0.001)$ and elevated TPOAb $(17.2 \%$ vs. $8.8 \%$ in controls; $P=0.01)$. The mean TSH level in controls was significantly higher than that in MMD (2.46 vs. $1.81 ; P=0.0007)$.

\section{Elevated thyroid function and autoantibodies in adult MMD}

A total of 169 patients were enrolled in this study. Of these, 91 (53.9\%) MMD patients presented with stroke (ischemic stroke, $n=54,32.0 \%$; hemorrhage stroke, $n=37,21.9 \%$ ). Smoking rate was more frequently observed in MMD with ischemic stroke than that with hemorrhage stroke $(P=0.013)$. Other variables such as hypertension, hyperlipidemia and coronary heart disease did not differ significantly among the three groups. Hyperthyroid status was more evident in MMD with ischemic presentation ( $n=$ $12,22.2 \%)$ compared to MMD without stroke $(n=$ $8,10.3 \%)$ or MMD with hemorrhage stroke $(n=0)$. Among autoantibodies, ischemic stroke was more frequently observed with elevated TPOAb $(25.9 \%)$ or TRAb $(13.0 \%)$ compared to MMD without stroke or MMD with hemorrhage stroke. Detailed comparisons of the three groups are shown in Table 2.

\section{Penalized multinomial logistic regression analysis for factors affecting stroke}

Due to the multicollinearity between thyroid function status and autoantibodies, two models were made. After a stepwise selection process including variables $(P<0.20)$, coronary heart disease and thyroid function status were selected for model 1 (Table 3). Hyperthyroidism had a marginally increased risk of MMD with ischemic stroke, with MMD without stroke as the reference value (OR, 2.53; 95\% CI: 0.979-6.822; $P=0.055$ ). Two factors of elevated $\mathrm{TPOAb}$ and coronary heart disease were chosen for model 2 after adjusting covariates. TPOAb increased the risk of MMD with ischemic stroke significantly (OR, 2.99; 95\% CI: 1.189-7.928; $P=0.020$ ). TRAb did not significantly increased the risk of MMD with ischemic stroke (OR, 2.03; $P=0.238)$.

\section{Meta-analysis of the association between thyroid abnormalities and adult MMD}

After reviewing literature, four studies, including ours, were selected for meta-analysis. ${ }^{7,8,14)} \mathrm{A}$ total of 241 MMD patients and 744 controls were included

Table 1 Comparison of patients with matched MMD and matched control from Ansung cohort

\begin{tabular}{lcccc}
\hline \multirow{2}{*}{ Variables } & MMD total & Matched MMD & Matched controls & \multirow{2}{*}{$P$-value } \\
\cline { 2 - 3 } & $(n=169)$ & $(n=116)$ & $(n=464)$ & \\
Age (years) & $40.6 \pm 11.6$ & $46.8 \pm 7.5$ & $47.3 \pm 6.9$ & \\
Female & $117(69.2 \%)$ & $82(70.7 \%)$ & $328(70.7 \%)$ & NA \\
T3 & $106.31 \pm 53.92$ & $101.02 \pm 20.36$ & NA & $<0.001$ \\
Free T4 & $1.20 \pm 0.50$ & $1.19 \pm 0.56$ & $1.00 \pm 0.14$ & 0.0007 \\
TSH & $1.81 \pm 1.91$ & $1.81 \pm 1.83$ & $2.46 \pm 2.07$ & $<0.001$ \\
Thyroid function status & & & & \\
Euthyroid & $142(84.0 \%)$ & $99(85.3 \%)$ & $404(87.1 \%)$ & NA \\
Hypothyroid & $7(4.1 \%)$ & $5(4.3 \%)$ & $54(11.6 \%)$ & NA \\
Hyperthyroid & $20(11.8 \%)$ & $12(10.3 \%)$ & $6(1.3 \%)$ & NA \\
Positive TGAb & $44(26 \%)$ & $30(25.8 \%)$ & NA & 0.01 \\
Positive TRAb & $12(7.1 \%)$ & $9(7.8 \%)$ & $21(8.8 \%)$ & \\
Positive TPOAb & $27(16.0 \%)$ & $20(17.2 \%)$ & & \\
\hline
\end{tabular}

MMD: moyamoya disease, TGAb: anti-thyroglobulin antibody, TRAb: thyrotropin receptor antibody, TPOAb: anti-thyroperoxidase antibody. 
Table 2 Comparison of clinical and laboratory characteristics among three groups in moyamoya disease

\begin{tabular}{|c|c|c|c|c|}
\hline \multirow{2}{*}{ Variables } & MMD without stroke & MMD with ischemic stroke & MMD with hemorrhagic stroke & \multirow{2}{*}{$P$-value } \\
\hline & $(n=78)$ & $(n=54)$ & $(n=37)$ & \\
\hline Age (years) & $39.7 \pm 11.4$ & $41.9 \pm 12.3$ & $40.68 \pm 11.03$ & 0.568 \\
\hline Female & $58(74.4 \%)$ & $32(59.3 \%)$ & $27(73.0 \%)$ & 0.155 \\
\hline Hypertension & $16(20.5 \%)$ & $21(38.9 \%)$ & $10(27.0 \%)$ & 0.068 \\
\hline Diabetes & $7(9.0 \%)$ & $3(5.6 \%)$ & $1(2.7 \%)$ & 0.478 \\
\hline Hyperlipidemia & $10(12.8 \%)$ & $11(20.4 \%)$ & $3(8.1 \%)$ & 0.230 \\
\hline Coronary heart disease & $1(1.3 \%)$ & $4(7.4 \%)$ & 0 & 0.112 \\
\hline Smoking & $15(19.2 \%)$ & $18(33.3 \%)^{\ddagger}$ & $3(8.1 \%)$ & 0.013 \\
\hline T3 & $101.1 \pm 18.9$ & $121.6 \pm 90.2^{*}$ & $94.8 \pm 16.7$ & 0.002 \\
\hline Free T4 & $1.2 \pm 0.7$ & $1.2 \pm 0.3$ & $1.2 \pm 0.3$ & 0.197 \\
\hline TSH & $2.0 \pm 2.2$ & $1.5 \pm 1.6$ & $1.9 \pm 1.6$ & 0.234 \\
\hline Thyroid function status & & & & 0.007 \\
\hline Euthyroid & $65(83.3 \%)$ & $41(75.9 \%)$ & $36(97.3 \%)$ & \\
\hline Hypothyroid & $5(6.4 \%)$ & $1(1.9 \%)$ & $1(2.7 \%)$ & \\
\hline Hyperthyroid & $8(10.3 \%)$ & $12(22.2 \%)^{*}$ & 0 & \\
\hline \multicolumn{5}{|l|}{ Elevated autoantibody } \\
\hline TGAb & $17(21.8 \%)$ & $17(31.5 \%)$ & $10(27.0 \%)$ & 0.454 \\
\hline TRAb & $5(6.4 \%)$ & $7(13.0 \%)^{\ddagger}$ & 0 & 0.045 \\
\hline TPOAb & $8(10.3 \%)$ & $14(25.9 \%)^{*}$ & $5(13.5 \%)$ & 0.049 \\
\hline
\end{tabular}

TGAb: anti-thyroglobulin antibody, TRAb: thyrotropin receptor antibody, TPOAb: anti-thyroperoxidase antibody, ${ }^{*} P<0.05$ versus both non-stroke and hemorrhagic group, ${ }^{\dagger} P<0.05$ versus non-stroke group, ${ }^{\ddagger} P<0.05$ versus hemorrhagic group.

Table 3 Penalized multinomial logistic regression analysis of factors affecting stroke in patients with moyamoya disease (MMD), MMD without stroke serving as a reference value

\begin{tabular}{|c|c|c|c|c|c|}
\hline & \multirow{2}{*}{ Variables } & \multicolumn{2}{|c|}{ MMD with hemorrhagic stroke } & \multicolumn{2}{|c|}{ MMD with ischemic stroke } \\
\hline & & OR (95\% CI) & $P$-value & OR (95\% CI) & $P$-value \\
\hline \multirow[t]{5}{*}{ Model 1} & Coronary heart disease & $0.59(0.004-11.336)$ & 0.738 & $5.16(0.911-52.892)$ & 0.064 \\
\hline & Thyroid function status & & & & \\
\hline & Euthyroid & 1 & & 1 & \\
\hline & Hypothyroid & $0.48(0.048-2.545)$ & 0.413 & $0.47(0.047-2.475)$ & 0.395 \\
\hline & Hyperthyroid & $0.10(0.001-0.876)$ & 0.034 & $2.53(0.979-6.822)$ & 0.055 \\
\hline \multirow[t]{2}{*}{ Model 2} & Coronary heart disease & $0.69(0.005-13.331)$ & 0.820 & $4.72(0.813-48.865)$ & 0.085 \\
\hline & Positive TPOAb & $1.40(0.418-4.358)$ & 0.571 & $2.99(1.189-7.928)$ & 0.020 \\
\hline
\end{tabular}

Reference category: MMD patients without stroke serving as a reference, TPOAb: anti-thyroperoxidase antibody.

for comparison. The number of elevated thyroid autoantibodies was $68(28.2 \%)$ in MMD and 55 $(7.4 \%)$ in controls. Elevated autoantibodies were significantly related to the adult MMD (OR, 7.663; CI: 2.055-28.574; $P=0.002$ ). Regarding the association between hyperthyroidism and adult MMD, $196 \mathrm{MMD}$ patients and 544 controls from three studies were included in the analysis. The number of hyperthyroidism was $34(17.4 \%)$ in MMD and $7(1.3 \%)$ in controls. Hyperthyroidism also significantly associated with adult MMD (OR, 10.936; CI: 4.617-25.904; $P<0.001$ ) (Figs. 1A and 1B). In the publication bias for comparison between adult MMD and elevated autoantibodies, Egger's regression test showed an intercept of -0.45 (95\% CI, -27.23 to $26.32 ; t=$ 0.22 , df $=1$; and $P=0.86$ ( 2 tailed). For the rank correlation test, Kendall's tau was 0 with 2-tailed $P=1.00$. Regarding adult MMD and hyperthyroidism, Egger's regression test showed an intercept of 1.08 (95\% CI, -2.44 to $4.60 ; t=3.90, \mathrm{df}=1$; and $P=0.16$ 


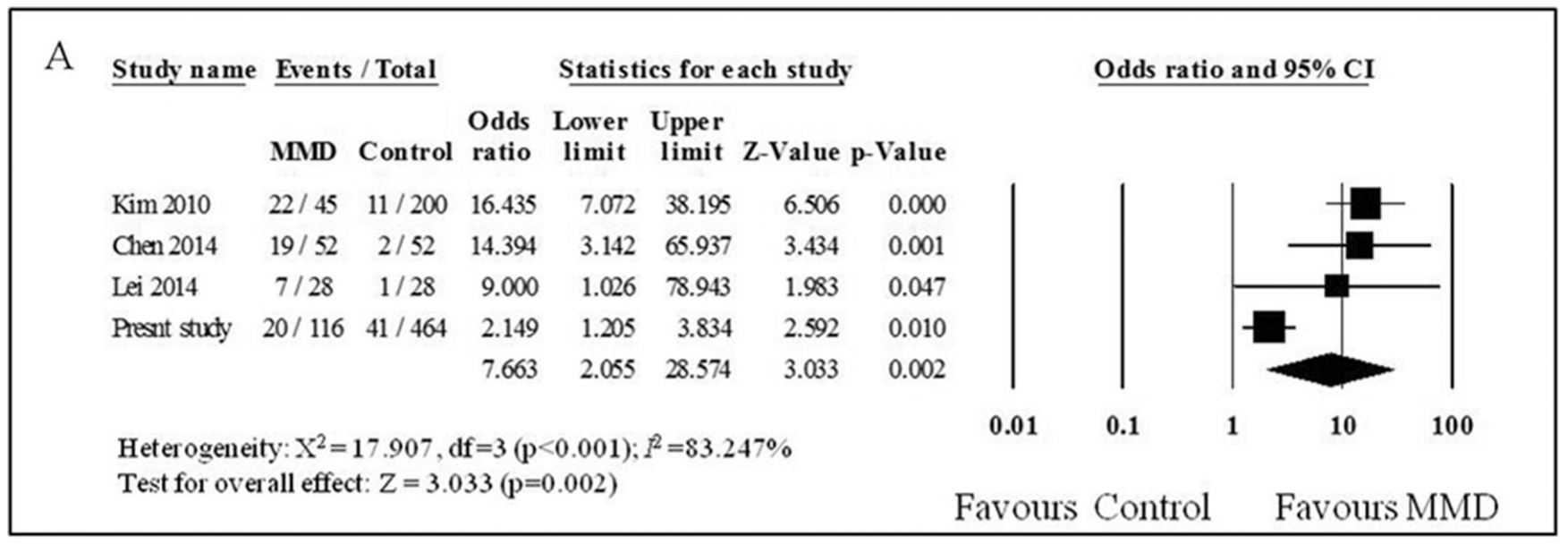

$\mathrm{B}$

Study name Events / Total

Statistics for each study

Odds ratio and $95 \% \mathrm{CI}$

\begin{tabular}{lccrrrrrr} 
& \multicolumn{2}{c}{$\begin{array}{r}\text { Odds } \\
\text { MMD }\end{array}$} & $\begin{array}{r}\text { Low trol } \\
\text { ratio }\end{array}$ & $\begin{array}{c}\text { Upper } \\
\text { limit }\end{array}$ & \multicolumn{2}{c}{ limit } & Z-Value p-Value \\
Chen 2014 & $17 / 52$ & $1 / 52$ & 24.771 & 3.150 & 194.776 & 3.051 & 0.002 \\
Lei 2014 & $5 / 28$ & $0 / 28$ & 13.340 & 0.701 & 253.894 & 1.724 & 0.085 \\
Presnt study & $12 / 116$ & $6 / 464$ & 8.808 & 3.231 & 24.010 & 4.252 & 0.000 \\
& & & 10.936 & 4.617 & 25.904 & 5.437 & 0.000
\end{tabular}

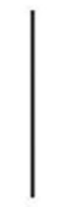

0.01

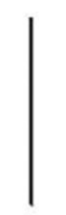

0.1

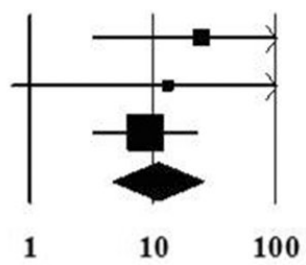

Heterogeneity: $\mathrm{X}^{2}=0.800, \mathrm{df}=2(\mathrm{p}=0.670) ; \not^{2}=0.000 \%$ Test for overall effect: $Z=5.437(p<0.001)$

\section{Favours Control Favours MMD}

Fig. 1 Comparisons of association between adult moyamoya disease (MMD) and elevated thyroid autoantibodies (A) and hyperthyroidism (B).

( 2 tailed). For the rank correlation test, Kendall's tau was 0.67 with 2-tailed $P=0.30$. Funnel plot analysis did not show bias in the included studies. Accordingly, there was no evidence of publication bias in these comparisons.

\section{Discussion}

We found a high prevalence of elevations of thyroid function and thyroid autoantibodies in adult MMD, especially in ischemic MMD. Although a genetic role and immunologic basis has been suggested for the pathogenesis of MMD, ${ }^{19,20)}$ the explanation for the different rate of the status of thyroid function and autoantibodies between different ethnic groups (Asian vs Caucasian) and the type of MMD (ischemic vs hemorrhagic) remains unclear. Furthermore, the clinical significances of the elevations of thyroid function and thyroid autoantibodies in patients with MMD are still unknown in adults. Our study showed that hyperthyroidism provided a marginally increased risk of MMD with ischemic stroke.
In particular, TPOAb was significantly related to MMD with ischemic stroke. Several mechanisms of elevated thyroid function and autoantibodies in adult MMD with ischemic stroke can be explained. Higher level of thyroid hormone can enhance sympathetic nervous activity which could lead to the abnormal vessel formation and vasoconstriction. T-cell dysfunction may induce vascular dysregulation which can lead to proliferation of the arterial wall. ${ }^{3,7)}$ Colleran et al. ${ }^{21)}$ reported that thyrotoxicosis may be associated with hyperhomocysteinemia and subsequent stroke. Aberrancies in thyroid autoimmunity may contribute to arterial narrowing and subsequent ischemic condition. Zhang et al. ${ }^{22)}$ reported a higher incidence of elevated TPOAb in stroke patients with intracranial stenosis without significant differences in thyroid function compared to those without intracranial stenosis. For Hashimoto's thyroiditis patient with euthyroidism, flow-mediated dilation was negatively correlated with TPOAb, which suggests an association between thyroid autoimmunity and impairment of endothelium-dependent 
arterial dilation. ${ }^{23)}$ Cardioembolic source by atrial fibrillation (AF) during thyrotoxicosis period also could attribute to ischemic stroke. ${ }^{24)}$ A prevalence rate of $10-15 \%$ of $\mathrm{AF}$ was noted in patients with hyperthyroidism. ${ }^{24)}$ Accordingly, effect of paroxysmal AF on cerebral infarct in adult MMD with abnormal thyroid condition should be studied further.

After reviewing the literature on the association between MMD and thyroid abnormalities, several concerns were identified especially to assess thyroid abnormalities in adult MMD patients. ${ }^{7,8,14)}$ First, difference between MMD and atherosclerotic steno-occlusive lesion should be considered for adults because atherosclerotic change in the arterial wall can develop as year-progress in time. In addition, secondary collateral moyamoya-like vessels can develop in patients with atherosclerotic steno-occlusive disease. ${ }^{25)}$ Accordingly, existing angiographic or magnetic resonance (MR) criteria for MMD could include the patients with MMD and atherosclerotic steno-occlusive disease together in determining risk factor of specific thyroid condition or thyroid autoantibodies in true MMD patients. In our department, we define MMD according to the specific diagnostic criteria to low the possibility of selection bias. ${ }^{11)}$ In particular, patients with risk factors of atherosclerosis (dyslipidemia, heavy smoking, alcoholics and hypertension) more than three were excluded in defining MMD patients. ${ }^{11,17)}$ Therefore, we think that our result could be derived from a more accurate method to estimate risk factors in adult MMD with thyroid abnormalities. Second, the distinction between definitive MMD and quasiMMD with underlying autoimmune disease such as Graves disease is not clear when the angiographic and clinical features are fully compatible with those of MMD. ${ }^{6,26)}$ Although we do not rule out the possibility that the population in this study could include the patients with quasi-MMD, the incidental coexistence of MMD and other autoimmune disease cannot be denied. Furthermore, autoimmune mechanism could be one of the strong candidates of the underlying pathology of MMD, which may obscure the distinction between MMD and quasi-MMD. ${ }^{6,27)}$ The advanced research in genetics and immunity to clarify the etiology of MMD is needed in the future. Third, analysis of the association, including typical bilateral and unilateral adult MMD together might cause biased conclusion. Although the contralateral angiographic progression of unilateral MMD can occur with a range of $0-50 \%,{ }^{9)}$ pathogenesis of the bilateral and unilateral MMD may be different. Jeon et al. ${ }^{28)}$ reported higher level of cellular retinoic acidbinding protein-I, which is a candidate protein for MMD pathogenesis, in bilateral MMD than unilateral MMD. Houkin et al. ${ }^{29)}$ also showed increased level of basic fibroblast growth factor in bilateral MMD than that in unilateral MMD. Accordingly, we only included the adult MMD patients who had bilateral angiographic MMD characteristics. Fourth, heterogeneous population mixed with pediatric and adults could make it difficult to identify the clinical significance of the specific thyroid condition and thyroid autoantibodies in MMD patients. Because the brain plasticity and initial presenting symptoms (hemorrhage or ischemia) are different between adult and pediatric $\mathrm{MMD},{ }^{28,30)}$ a previous meta-analysis ${ }^{8)}$ could not fully reflect the association between adult MMD and elevated thyroid condition and autoantibodies. Therefore, we only included adult MMD patients for the meta-analysis.

The rate of hyperthyroid status and elevated TPOAb in adult MMD of this study was $11.8 \%$ and $16 \%$, respectively. The rates seen on our study was in accordance with the range of 10.5-17.7\% or $11.4-26.7 \%$ in the previous reports., ${ }^{3,7)}$ On the contrary, $1.3 \%$ of hyperthyroid status and $8.8 \%$ of elevated TPOAb in Anusng cohort was higher than those in healthy controls in the previous reports with range of $0.9 \%$ and $1.8-5.5 \%$, respectively. ${ }^{31}$ Although prevalence rate of hyperthyroid status and elevated TPOAb was relatively high in Ansung cohort, we think that our control cohort, which was based on large population, might be well representative of the general population. ${ }^{31)}$ Therefore, our results might accurately reflect the association between adult MMD and thyroid abnormalities than a hospital-based case-control study.

There are some limitations in this study. First, we only checked thyroid laboratory tests at a single time point, especially at the time of admission. Because initial laboratory findings do not exactly reflect clinical status in ischemic stroke, serial verifications of thyroid hormone and autoantibodies are necessary including at the time of clinical events to strengthen our results. Second, our meta-analysis could have limited value in Asian MMD patients due to the absence of other ethnicities' data. Third, heterogeneity across studies, in particular elevated thyroid autoantibody and MMD, can be a concern. We think that difference in control groups (hospital-based control vs. community-based control), outcome measure of thyroid abnormalities, and type of adult MMD patients (typical bilateral vs. probable MMD) could affect substantial heterogeneity in the studies; however, remaining analysis showed minimal heterogeneity.

\section{Conclusions}

Elevated TPOAb and hyperthyroidism may play important roles in the development of adult MMD 
with ischemic stroke, which could be used for screening of targeted hyperthyroidism and thyroid autoantibodies in the future. Prospective large studies, including various ethnicities are needed to confirm our results.

\section{Acknowledgments}

This work was supported by a National Research Foundation of Korea (NRF) grant funded by the Ministry of Science, Information and Communication Technologies (ICT) and Future Planning (MSIP) of the Korea Government (No. 2017M3A9E8033223). This study was also supported by the Seoul National University Hospital (No. 0620142800).

\section{Conflicts of Interest Disclosure}

None.

\section{References}

1) Suzuki J, Takaku A: Cerebrovascular "moyamoya” disease. Disease showing abnormal net-like vessels in base of brain. Arch Neurol 20: 288-299, 1969

2) Im SH, Oh CW, Kwon OK, Kim JE, Han DH: Moyamoya disease associated with Graves disease: special considerations regarding clinical significance and management. J Neurosurg 102: 1013-1017, 2005

3) Li H, Zhang ZS, Dong ZN, et al.: Increased thyroid function and elevated thyroid autoantibodies in pediatric patients with moyamoya disease: a casecontrol study. Stroke 42: 1138-1139, 2011

4) Squizzato A, Gerdes VE, Brandjes DP, Büller HR, Stam J: Thyroid diseases and cerebrovascular disease. Stroke 36: 2302-2310, 2005

5) Bower RS, Mallory GW, Nwojo M, Kudva YC, Flemming $\mathrm{KD}$, Meyer FB: Moyamoya disease in a primarily white, midwestern US population: increased prevalence of autoimmune disease. Stroke 44: 1997-1999, 2013

6) Fujimura M, Tominaga T: Diagnosis of moyamoya disease: international standard and regional differences. Neurol Med Chir (Tokyo) 55: 189-193, 2015

7) Kim SJ, Heo KG, Shin HY, et al.: Association of thyroid autoantibodies with moyamoya-type cerebrovascular disease: a prospective study. Stroke 41: 173-176, 2010

8) Lei C, Wu B, Ma Z, Zhang S, Liu M: Association of moyamoya disease with thyroid autoantibodies and thyroid function: a case-control study and metaanalysis. Eur J Neurol 21: 996-1001, 2014

9) Lee SC, Jeon JS, Kim JE, et al.: Contralateral progression and its risk factor in surgically treated unilateral adult moyamoya disease with a review of pertinent literature. Acta Neurochir (Wien) 156: 103-111, 2014
10) Scott RM, Smith ER: Moyamoya disease and moyamoya syndrome. N Engl J Med 360: 1226-1237, 2009

11) Kim JE, Jeon JS: An update on the diagnosis and treatment of adult moyamoya disease taking into consideration controversial issues. Neurol Res 36: 407-416, 2014

12) Kleinloog R, Regli L, Rinkel GJ, Klijn CJ: Regional differences in incidence and patient characteristics of moyamoya disease: a systematic review. J Neurol Neurosurg Psychiatry 83: 531-536, 2012

13) Shin C, Abbott RD, Lee H, Kim J, Kimm K: Prevalence and correlates of orthostatic hypotension in middle-aged men and women in Korea: the Korean Health and Genome Study. J Hum Hypertens 18: 717-723, 2004

14) Chen JB, Lei D, He M, Sun H, You C, Zhou LX: Association of thyroid autoantibodies and thyroid function with moyamoya disease: a prospective study in Western China. J Neurol Sci-Turkish 31: 709-717, 2014

15) Miyamoto S, Yoshimoto T, Hashimoto N, et al.; JAM Trial Investigators: Effects of extracranial-intracranial bypass for patients with hemorrhagic moyamoya disease: results of the Japan Adult Moyamoya Trial. Stroke 45: 1415-1421, 2014

16) Qian C, Yu X, Li J, Chen J, Wang L, Chen G: The efficacy of surgical treatment for the secondary prevention of stroke in symptomatic moyamoya disease: a meta-analysis. Medicine (Baltimore) 94: e2218, 2015

17) Jeon JP, Kim JE: A recent update of clinical and research topics concerning adult moyamoya disease. J Korean Neurosurg Soc 59: 537-543, 2016

18) Egger M, Davey Smith G, Schneider M, Minder C: Bias in meta-analysis detected by a simple, graphical test. BMJ 315: 629-634, 1997

19) Achrol AS, Guzman R, Lee M, Steinberg GK: Pathophysiology and genetic factors in moyamoya disease. Neurosurg Focus 26: E4, 2009

20) Uchino K, Johnston SC, Becker KJ, Tirschwell DL: Moyamoya disease in Washington State and California. Neurology 65: 956-958, 2005

21) Colleran KM, Ratliff DM, Burge MR: Potential association of thyrotoxicosis with vitamin B and folate deficiencies, resulting in risk for hyperhomocysteinemia and subsequent thromboembolic events. Endocr Pract 9: 290-295, 2003

22) Zhang X, Chen Z, Shi Z, Lou M: Correlation between thyroid autoantibodies and intracranial arterial stenosis in stroke patients with hyperthyroidism. J Neurol Sci 318: 82-84, 2012

23) Xiang GD, He YS, Zhao LS, Hou J, Yue L, Xiang HJ: Impairment of endothelium-dependent arterial dilation in Hashimoto's thyroiditis patients with euthyroidism. Clin Endocrinol (Oxf) 64: 698-702, 2006

24) Klein I, Ojamaa K: Thyroid hormone and the cardiovascular system. $N$ Engl J Med 344: 501-509, 2001

25) Jiang T, Perry A, Dacey RG, Zipfel GJ, Derdeyn CP: Intracranial atherosclerotic disease associated with moyamoya collateral formation: histopathological findings. J Neurosurg 118: 1030-1034, 2013 
26) Takahashi JC, Miyamoto S: Moyamoya disease: recent progress and outlook. Neurol Med Chir (Tokyo) 50: 824-832, 2010

27) Research committee on the pathology and treatment of spontaneous occlusion of the circle of willis; health labour sciences research grant for research on measures for infractable diseases: Guidelines for diagnosis and treatment of moyamoya disease (spontaneous occlusion of the circle of willis). Neurol Med Chir (Tokyo) 52: 245-266, 2012

28) Jeon JS, Ahn JH, Moon YJ, et al.: Expression of cellular retinoic acid-binding protein-I (CRABP-I) in the cerebrospinal fluid of adult onset moyamoya disease and its association with clinical presentation and postoperative haemodynamic change. J Neurol Neurosurg Psychiatry 85: 726-731, 2014

29) Houkin $K$, Abe $H$, Yoshimoto $T$, Takahashi A: Is "unilateral" moyamoya disease different from moyamoya disease? J Neurosurg 85: 772-776, 1996

30) Kuroda S, Ishikawa T, Houkin K, Nanba R, Hokari M, Iwasaki Y: Incidence and clinical features of disease progression in adult moyamoya disease. Stroke 36: 2148-2153, 2005

31) Cho NH, Choi HS, Kim KW, et al.: Interaction between cigarette smoking and iodine intake and their impact on thyroid function. Clin Endocrinol $(O x f)$ 73: 264-270, 2010

Address reprint requests to: Jeong Eun Kim, MD, PhD, Department of Neurosurgery, Seoul National University College of Medicine, 101 Daehak-ro, Jongno-gu, Seoul 110-744, Korea.

e-mail: eunkim@snu.ac.kr 